\title{
Uma Aproximação para o Problema de Alocação de Terminais com Capacidade
}

\author{
Lehilton L.C. Pedrosa, Vinícius Balbino de Souza ${ }^{\dagger}$ \\ ${ }^{1}$ Instituto de Computação - Universidade Estadual de Campinas \\ Campinas, São Paulo \\ lehilton@ic.unicamp.br, vbalbinods@gmail.com
}

\begin{abstract}
We consider the Capacitated p-Hub Center Problem. An instance comprises a metric space $V$, a set of demands $D \subseteq V^{2}$, a number of hubs $p$, and a capacity $L$. A solution is a multiset $S$ of locations where to install hubs with $|S| \leq p$ and an assignment from each demand to a hub such that no hub receives more than L demands. The objective is to find the solution that minimizes the maximum cost of serving a demand through the assigned hub. In this work, we give the first approximation algorithm for the problem, that achieves factor 7 .
\end{abstract}

Resumo. Consideramos o Problema de Alocação de Terminais com Capacidade. Uma instância é composta de um espaço métrico $V$, um conjunto de demandas $D \subseteq V^{2}$, um número de terminais p e uma capacidade L. Uma solução é um multiconjunto $S$ de locais para instalar terminais com $|S| \leq p$ e, para cada demanda, um terminal associado de forma que nenhum deles receba mais de $L$ demandas. O objetivo é encontrar uma solução que minimiza o maior custo de servir uma demanda através do terminal atribuído. Neste trabalho, obtemos o primeiro algoritmo de aproximação para o problema, com fator 7 .

\section{Introdução}

Em diversas aplicações de transportes, logística, etc., necessitamos da instalação de terminais, como aeroportos de conexão ou switches de rede. Um terminal é um centro de consolidação, troca e ordenação, que permite remanejamento de conexões diretas, usando uma quantidade menor de ligações indiretas. Uma vez que a maioria de problemas voltados a essas aplicações são NP-difíceis, consideramos alternativas, como algoritmos de aproximação: um algoritmo polinomial para um problema de otimização é uma $\alpha$-aproximação se o custo da solução encontrada é no máximo $\alpha$ vezes mais caro que a melhor solução existente. Enquanto aproximações para problemas de localização, como o problema clássico dos $k$-Centros são conhecidas desde os anos 80 [Hochbaum and Shmoys 1986], as primeiras aproximações para problemas de alocação de terminais só apareceram depois, como para o chamado Problema de Alocação de Terminais em Estrela, em 2009 [Iwasa et al. 2009]. Recentemente, Pedrosa et al. [Pedrosa et al. 2016] consideraram o Problema de Centro de Alocação dos p-Terminais (Capacitated p-Hub Center Problem, $p$-HP), mostrando que é NP-difícil encontrar uma aproximação com fator melhor que 2 e obtendo um algoritmo com fator 3.

\footnotetext{
*Este trabalho foi parcialmente financiado pelo processo no 2015/11937-9, Fundação de Amparo à Pesquisa do Estado de São Paulo (FAPESP).

${ }^{\dagger}$ Bolsista do CNPq - Brasil.
} 
Um exemplo de situação modelada pelo $p$-HP ocorre se quisermos conectar pares de computadores instalando um número $p$ de roteadores. Nesse caso, o objetivo é minimizar a pior latência entre um computador de origem, o roteador associado e o computador de destino. Na prática, um roteador pode ter um limite de conexões a que pode atender, o que significa que uma solução para o $p$-HP nem sempre é aplicável. Neste trabalho, consideramos uma generalização do $p$-HP para modelar esse tipo de restrição. Especificamente, consideramos o Capacitated $p$-HP, denotado por $p$-CHP, em que uma entrada também contém um número $L$ e cada um dos $p$ terminais instalados pode ser associado a no máximo $L$ demandas.

Nossa contribuição é a primeira aproximação para o $p$-CHP, com fator de aproximação 7. O algoritmo utiliza o método do gargalo [Hochbaum and Shmoys 1986], em que se reduz um problema de um espaço métrico para o caso particular em que os pontos estão em um grafo sem pesos. Para lidar com as capacidades, criamos um particionamento de demandas e resolvemos um problema de fluxo máximo, baseando-se na árvore de monarcas de [Khuller and Sussmann 2000], utilizada em sua clássica 5-aproximação para o problema dos $k$-centros com capacidade.

\section{Uma 7-Aproximação para o $p$-CHP}

Formalmente, uma instância do $p$-CHP é dada por um grafo completo $G$ com vértices $V$ e métrica $d$, em que $d(x, y) \geq 0$ é a distância entre os vértices $x, y \in V$; um conjunto $D$ de pares $(x, y) \in V \times V$, em que cada um representa uma demanda entre os vértices $x$ e $y$; um número inteiro $p$, que define o número de terminais a serem instalados; e um número $L$, cada um representando' a capacidade de um terminal. Uma solução é um multiconjunto $S$ de elementos de $V$, com $|S| \leq p$, e uma função $\phi: D \rightarrow S$, tal que $\left|\phi^{-1}(v)\right| \leq L$ para todo $v \in S$ (i.e., $\phi$ associa no máximo $L$ demandas a $v$ ). O objetivo é encontrar uma solução que minimiza $\max _{(x, y) \in D} d(x, \phi(x, y))+d(\phi(x, y), y)$.

\subsection{Algoritmo}

$\mathrm{O}$ algoritmo para o $p$-CHP consiste de quatro rotinas, $p$-CAPHUB, GETMONARCHS, SETDomains e Assign, que são detalhadas nos Algoritmos 1 a 4. Uma descrição textual é apresentada a seguir.

A rotina $p$-CAPHUB obtém uma estimativa do valor de uma solução ótima, $\tau$, enumerando cada uma das possibilidades. Dada uma estimativa $\tau$, cria-se um grafo $H$ bipartido entre demandas $D$ e locais $V$, chamado de grafo de gargalo, em que uma aresta significa que uma demanda pode ser servida a partir de um certo local ao custo de no máximo $\tau$. Se houver demanda isolada, então pode-se testar o próximo valor de $\tau$ no conjunto de possibilidades, já que nesse caso não existe terminal que satisfaz essa demanda com custo $\tau$. Se toda demanda é incidente a pelo menos uma aresta em $H$, então o problema se reduz em encontrar uma solução com o número mínimo de terminais em que cada demanda é satisfeita por um terminal na vizinhança de $H$. Iremos mostrar depois que, para um certo $\tau$, ou $p$-CAPHUB encontra uma solução com até $p$ terminais em que cada demanda é atribuída a um terminal à distância até 7 em $H$, ou em qualquer solução do problema original de custo até $\tau$, mais do que $p$ terminais são necessários. No primeiro caso, o algoritmo devolverá uma 7-aproximação e o algoritmo termina; no último caso, conclui-se que $\tau$ está subestimando o valor ótimo, e testa-se a próxima possibilidade. 


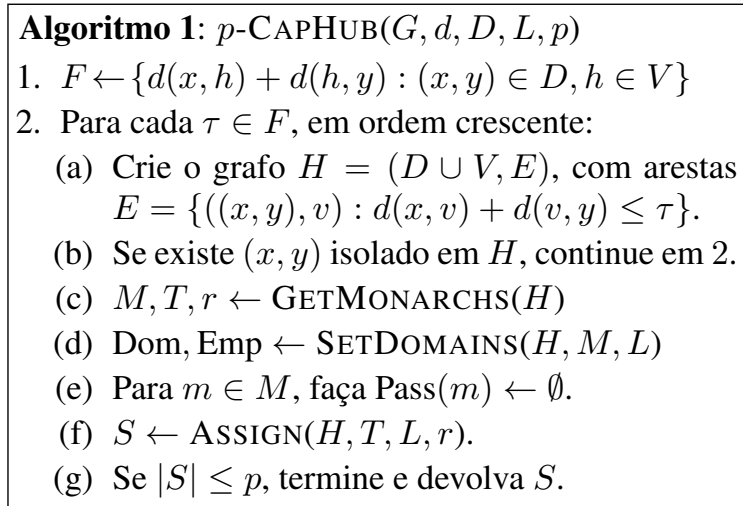

Algoritmo 3: $\operatorname{SetDomains}(H, M, L)$

1. Crie uma rede de fluxo $N$ com vértices $D$, uma cópia de cada demanda de $M$ e dois novos nós, $s, t$. O conjunto de arcos é:

- Para $e \in D$, um arco $(s, e)$ de capacidade 1 .

- Para $m \in M$, um $\operatorname{arco}(m, t)$ de capacidade $L$.

- Para cada $e \in D$ e cópia $m \in M$, um arco não capacitado $(e, m)$ se $\operatorname{dist}_{H}(e, m) \leq 2$.

2. Obtenha um fluxo $s$-t máximo em $N$.

3. Para cada $m \in M$, seja $\operatorname{Dom}(m)$ (domínio de $m$ ) o conjunto de demandas $e$ por que passa uma unidade de fluxo e cujo fluxo flua por $m$.

4. Para cada $m$, seja $\operatorname{Emp}(m)$ (império de $m$ ) o conjunto de demandas $e$ com $d(m, e) \leq 2$ que ainda não estão no domínio ou império de algum monarca. Devolva Dom e Emp.

\author{
Algoritmo 2: GetMonarchs $(H)$ \\ 1. Escolha demanda $r \in D$ arbitrária. \\ 2. Faça $M \leftarrow\{r\}$ e torne $r$ raiz de $T$. \\ 3. Enquanto houver $e \in D \backslash M$, $\operatorname{dist}_{H}(e, M) \geq 4$ : \\ (a) Escolha $f \in M$ e $e \in D \backslash M$, tais que \\ $\operatorname{dist}_{H}(e, f)=4$. \\ (b) Adicione $e$ a $M$ e faça $f$ pai de $e$ em $T$.
}

4. Devolva $M, T$ e $r$.

\section{Algoritmo 4: $\operatorname{Assign}(H, T, L, m)$}

1. Execute $\operatorname{Assign}(H, T, L, f)$ em cada filho $f$ de $m$.

2. Sejam $b \geq 0$ e $0 \leq c<L$ números inteiros tais que $b L+c=|\operatorname{Pass}(m)|+|\operatorname{Emp}(v)|$.

3. Escolha um vizinho arbitrário $v$ de $m$ no grafo $H$ e instale $b+1$ terminais em $v$.

4. Atribua cada demanda $e \in \operatorname{Pass}(m) \cup \operatorname{Emp}(m)$ aos $b+1$ terminais; em seguida, atribua a esses quantos elementos de $\operatorname{Dom}(v)$ forem possíveis sem exceder a capacidade.

5. Se $m$ for a raiz:

- então instale um novo terminal em $v$ e atribua as demandas excedentes de $\operatorname{Dom}(m)$;

6. Caso contrário:

- inclua as demandas excedentes em $\operatorname{Pass}\left(m^{\prime}\right)$, em que $m^{\prime}$ é o pai de $m$.

7. Devolva o conjunto de terminais instalados.

O laço interno de $p$-CAPHUB contém três chamadas a sub-rotinas. A primeira, GETMONARCHS, obtém a chamada árvore de monarcas, $T$, de maneira gulosa. $T$ é construída de forma que monarcas pai e filho estejam à distância $4 \mathrm{em} H$ e que cada demanda $e \in D$ seja um monarca, ou esteja a distância 2 de algum monarca, i.e, $\operatorname{dist}_{H}(e, m) \leq 2$ para $m \in M$. Essa propriedade garante que, para cada monarca $m \in M$, uma solução ótima $S^{*}$ contenha um terminal $h \in S^{*}$, chamado testemunha, que é vizinho de $m$ em $H$.

A segunda sub-rotina é SetDomains. Para cada $m \in M$, definem-se conjuntos disjuntos $\operatorname{Dom}(m)$ e $\operatorname{Emp}(m)$ de demandas com distância até 2 de $m$, chamados de domínio e império de $m$. A família de todos pares $\operatorname{Dom}(m)$ e $\operatorname{Emp}(m)$ particionam $D$. Os domínios são escolhidos de forma que $|\operatorname{Dom}(m)| \leq L$ para cada $m$, mas de forma a maximizar o número de demandas que podem ser servidas se houvesse um terminal em cada monarca. Intuitivamente, a união dos domínios serve pelo menos tantas demandas quantas são servidas na solução ótima $S^{*}$ pelas testemunhas de $M$. Para maximizar o número de demandas servidas pelos monarcas, é resolvido um problema de fluxo máximo.

Finalmente, ASSIGN instala e atribui demandas a terminais, percorrendo a árvore de monarcas de forma bottom-up. Para cada monarca $m$, escolhe-se um local $v$ na vizinhança de $m$ e instalam-se $b+1$ terminais, a fim de servir as demandas de $\operatorname{Emp}(m)$ e, possivelmente, um conjunto de demandas passadas pelos filhos de $m$, $\operatorname{Pass}(m)$. As demandas em $\operatorname{Dom}(m)$ são atribuídas aos terminais com capacidade livre em $v$ e as excedentes passadas ao pai de $m$. O algoritmo termina quando a raiz é considerada. Nesse caso, não é possível transferir demandas remanescentes e um novo terminal é instalado. 


\subsection{Análise}

A correção do algoritmo utiliza dois fatos principais: para um dado $\tau$, a rotina $p$-CAPHUB não seleciona mais terminais do que uma solução ótima; e, para cada demanda, o terminal associado está à distância no máximo 7. Primeiro precisamos da seguinte definição.

Definição. Um monarca leve é um monarca $m \in M \operatorname{com}|\operatorname{Dom}(m)|<L$. Um monarca cheio é um monarca $m \in M$ com $|\operatorname{Dom}(m)|=L$.

Lema 1. Seja $k_{L}$ o número de monarcas leves, $n_{L}$ é o número de demandas nos domínios de monarcas leves e n o número total de demandas. Se $S^{*}$ é o conjunto de terminais de uma solução de custo $\tau$ com um número mínimo de terminais, então $\left|S^{*}\right| \geq k_{L}+\left\lceil\frac{n-n_{L}}{L}\right\rceil$.

O seguinte lema limita a distância entre uma demanda e o terminal atribuído.

Lema 2. Seja e uma demanda. Se e foi atribuída a um terminal $v$, então $\operatorname{dist}_{H}(e, v) \leq 7$.

Demonstração. Seja $e$ uma demanda e seja $m$ o monarca correspondente à execução de ASSIGN no momento em que $e$ foi atribuída. Vamos mostrar que dist $\operatorname{di}_{H}(e, m) \leq 6$. Consideramos dois casos. Se $e$ pertence ao domínio ou ao império de $m$, então temos $\operatorname{dist}_{H}(e, m) \leq 2$. Do contrário, então $e \in \operatorname{Pass}(m)$, i.e., $e$ é uma demanda passada por um filho de $m$ na árvore de monarcas, diga-se $m^{\prime}$. Como $e$ não foi atribuído a $m^{\prime}$, segue que $e \in \operatorname{Dom}\left(m^{\prime}\right)$. Obtemos $\operatorname{dist}_{H}\left(m^{\prime}, m\right)=4$ e $\operatorname{dist}_{H}\left(e, m^{\prime}\right) \leq 2$. Combinando essas equações, obtemos $\operatorname{dist}_{H}(e, m) \leq \operatorname{dist}_{H}\left(e, m^{\prime}\right)+\operatorname{dist}_{H}\left(m^{\prime}, m\right) \leq 2+4=6$.

Para concluir o lema, basta observar que todas as demandas atribuídas por ASSIGN, ao ser executado com monarca $m$, são atribuídas a um mesmo local $v$ na vizinhança de $m$. Daí, $\operatorname{dist}_{H}(m, v)=1 \mathrm{e}$, finalmente, $\operatorname{dist}_{H}(e, v) \leq \operatorname{dist}_{H}(e, m)+$ $\operatorname{dist}_{H}(m, v) \leq 6+1=7$.

Lema 3. Seja $v \in V$ e e $=(x, y) \in D$. Se $\operatorname{dist}_{H}(e, v)=\alpha$, então $d(x, v)+d(v, y) \leq \alpha \tau$.

Teorema 1. Existe uma 7-aproximação para o p-CHP.

Demonstração. Primeiro note que o algoritmo executa em tempo polinomial. Também, pelo Lema 2, em cada iteração de $p$-CAPHUB com valor $\tau$, obtemos uma atribuição $\phi$ tal que para cada $e \in D$, $\operatorname{dist}_{H}(e, \phi(e)) \leq 7$. Pelo Lema 3 , obtemos que $d(e, \phi(e)) \leq 7 \tau$.

Seja OPT o custo da solução ótima $S^{*}$. Note que OPT é considerado pelo algoritmo $p$-CAPHUB, i.e., OPT $\in F$. Pelo Lema 1 , na iteração em que $\tau=\mathrm{OPT}$, vale $\left|S^{*}\right| \geq k_{L}+\left\lceil\frac{n-n_{L}}{L}\right\rceil$. Como os únicos terminais instalados e não completamente ocupados estão associados a monarcas leves e, possivelmente, um único terminal instalado para a raiz $r$, segue que o ASSIGN instala, no máximo, $k_{L}+\left\lceil\frac{n-n_{L}}{L}\right\rceil \leq\left|S^{*}\right| \leq p$ terminais. Portanto, para alguma iteração com valor $\tau^{\prime} \leq$ OPT, o algoritmo irá devolver uma solução de custo no máximo $7 \tau^{\prime}$ e com no máximo $p$ terminais.

\section{Referências}

[Hochbaum and Shmoys 1986] Hochbaum, D. S. and Shmoys, D. B. (1986). A Unified Approach to Approximation Algorithms for Bottleneck Problems. J. ACM, 33(3):533-550.

[Iwasa et al. 2009] Iwasa, M., Saito, H., and Matsui, T. (2009). Approximation algorithms for the single allocation problem in hub-and-spoke networks and related metric labeling problems. Discrete Applied Mathematics, 157(9):2078-2088.

[Khuller and Sussmann 2000] Khuller, S. and Sussmann, Y. J. (2000). The Capacitated K-Center Problem. SIAM Journal on Discrete Mathematics, 13(3):403-418.

[Pedrosa et al. 2016] Pedrosa, L. L. C., Santos, V. F., and Schouery, R. C. S. (2016). Uma aproximação para o problema de alocação de terminais. In Anais do CSBC 2016 ( $1^{\circ}$ ETC - 2016). 